\title{
Asymmetric dimethylarginine (ADMA), symmetric dimethylarginine (SDMA) and L-arginine in patients with arteriogenic and non-arteriogenic erectile dysfunction
}

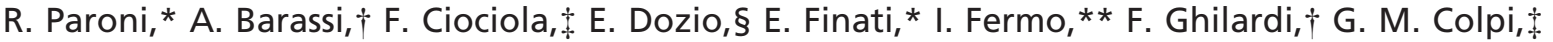 \\ M. M. Corsi§, $\uparrow$ and G. V. Melzi d’Eril† \\ *Unita' di Chimica e Biochimica Analitica, Dipartimento di Medicina, Chirurgia e Odontoiatria, Università degli Studi di Milano, Italy, †Laboratorio \\ di Analisi, Dipartimento di Medicina, Chirurgia e Odontoiatria, Ospedale San Paolo, Università degli Studi di Milano, Italy, \$Unità di Urologia \\ Andrologica, Ospedale San Paolo, Milano, Italy, §Dipartimento di Morfologia Umana e Scienze Biomediche "Città Studi", Facoltà di Medicina e \\ Chirurgia, Università degli Studi di Milano, Italy, -Unità di Patologia Clinica, IRCCS Policlinico San Donato, San Donato, Italy, and **Unita' di \\ Tecniche Separative, Istituto Scientifico San Raffaele, Milano, Italy
}

\begin{abstract}
Keywords:
arteriogenic erectile dysfunction, asymmetric dimethylarginin, non-arteriogenic erectile dysfunction, symmetric dimethylarginine
\end{abstract}

\section{Correspondence:}

Alessandra Barassi, MD, Dipartimento di Medicina, Chirurgia e Odontoiatria, Università degli Studi di Milano, Via di Rudinì 8, 20142 Milano, Italy.

E-mail: Alessandra.barassi@unimi.it

Received 31 August 2011; revised 10 February 2012; accepted 29 February 2012

doi:10.1111/j.1365-2605.2012.01272.x

\section{Summary}

The plasma concentration of asymmetrical dimethylarginine (ADMA), an inhibitor of nitric oxide synthase, has been linked to endothelial dysfunction. We investigated the relation between ADMA, symmetric dimethylarginine (SDMA) and L-arginine concentrations and erectile dysfunction. We compared plasma levels of ADMA, SDMA and L-arginine in 61 men in good health with erectile dysfunction of arteriogenic and non-arteriogenic origin. Diagnosis of erectile dysfunction was based on the International Index of Erectile Function Score and its aetiology was classified with penile echo-colour-Doppler in basal condition and after intracavernous injection of prostaglandin E1. The ADMA and SDMA concentrations were significantly higher in men with arteriogenic erectile dysfunction compared with those with erectile dysfunction of non-arteriogenic origin $(p<0.05)$ and the concentrations in both subgroups were significantly higher than in controls $(p<0.001)$. There was a negative correlation between ADMA and International Index of Erectile Function Score only in arteriogenic erectile dysfunction subgroup. L-arginine did not differ significantly neither between the two erectile dysfunction subgroups $(p>0.05)$ nor between each of the two erectile dysfunction subgroups and controls $(p>0.05)$. The L-arginine/ADMA and the L-arginine/SDMA ratios in arteriogenic erectile dysfunction subgroups were significantly lower than both in controls $(p<0.05)$ and in non-arteriogenic erectile dysfunction patients $(p<0.05)$; the two ratios in nonarteriogenic erectile dysfunction patients did not differ from those in the controls $(p>0.05)$. We conclude that ADMA and SDMA concentrations are significantly higher and L-arginine/ADMA ratio lower in patients who have arteriogenic erectile dysfunction compared with both patients with non-arteriogenic erectile dysfunction and controls. The negative correlation between ADMA and severity of erectile dysfunction is present only in patients with arteriogenic erectile dysfunction. This study supports the importance to always distinguish arteriogenic from non-arteriogenic erectile dysfunction patients to study the complicate erectogenic mechanisms that lead to erectile dysfunction and also to provide potential therapeutic agents for patients with arteriogenic erectile dysfunction.

\section{Introduction}

Erectile dysfunction (ED) is highly prevalent and by current estimated, 30 million men in US and 150 million men worldwide are affected and occurs in 19-64\% of men aged $40-80$ years, both developing and industrialized countries (Benet et al., 1995). Although ED is a multifactorial process, vascular disease of the penile arteries is an 
important cause of ED accounting for up to $80 \%$ of cases (Kendirci et al., 2007). It has been suggested that endothelial dysfunction is linked to ED as both share a dependence on a common pathway through the nitric oxide (NO) release (Burchardt et al., 2001; Hashimoto et al., 2000; Widlansky et al., 2003; De Angelis et al., 2001). NO is the major endothelium-derived relaxing factor, playing a critical role in the maintenance of vascular tone (Palmer et al.,1988). Penile tumescence and erection is critically reliant on the NO release by both cavernosal nerve terminals and endothelial cells (Sàenz de Tajada et al., 2004). $\mathrm{NO}$ is formed from the precursor amino acid, L-arginine, by enzymatic action of nitric oxide synthase (NOS). NO production may be regulated by endogenous NOS competitive inhibitor asymmetric dimethylarginine (ADMA), a L-arginine analogous. The ADMA levels in plasma are a balance between breakdown of proteins containing methylated arginine, renal excretion and the activity of hepatic dimethylarginine dimethylaminohydrolase (DDAH). The L-arginine analogous identified to day, include monomethylarginine (NMMA), symmetric dimethylarginine (SDMA) and ADMA. It was postulated that ADMA, which was identified as circulating in human plasma at a concentration 10 times greater than that of naturally occurring NMMA, might act as an important endogenous regulator of the NO pathway (Vallance et al., 1992).

In view of the apparent role of $\mathrm{NO}$ as the key chemical element necessary for penile erection, investigators have begun to explore the incidence of ED in conditions associated to a deregulated ADMA and/or SDMA metabolism as diabetes mellitus, hypercholesterolaemia, renal failure, hypogonadism, ageing and irradiation (Schiel et al., 2003; Maas et al., 2005; Elesberg et al., 2006; Wierzbicki et al., 2006; Kielstein et al., 2005; Lugg et al., 1995; Garban et al., 1995; Carrier et al., 1995). Moreover, over the past decade the interference of these substrates with cardiovascular function has received increasing interest and several lines of evidence suggest that ADMA is involved in the pathogenesis of cardiovascular diseases (Valkonen et al., 2001; Cooke et al., 2004).

As ED may be an early marker for the presence of subclinical atherosclerosis (Solomon et al., 2003) we studied the plasma concentrations of L-arginine, ADMA and SDMA in men with arteriogenic and non-arteriogenic $\mathrm{ED}$, to assess the differences between the two ED subgroups and the healthy subjects, independently by the presence of clinical or subclinical atherosclerosis.

\section{Materials and methods}

\section{Investigation protocol}

In our centre, patients complaining of ED are currently investigated by careful history-taking and clinical andro- logical examination; then, a few days later, by a panel of examinations, including blood tests, complete blood picture, haemoglobin, glycated haemoglobin, glycaemia, urea, creatinine, PCR, total and HDL cholesterol, triglycerides, transaminases, testosterone, prolactin, 17- $\beta$-estradiol, urine analysis and 24-h urinary albumin excretion (microalbuminuria), the International Index of Erectile Function questionnaire (IIEF) and echo-colour-Doppler of both cavernous arteries.

The IIEF questionnaire (Rosen et al., 1999) is a validated, self-administered tool (Rosen et al., 1999; Rosen et al., 1997), but we only evaluated the answers to the first five (erectile response dominium) of the 15 questions (IIEF-15, 1-5). Possible scores for the IIEF-5 range from 5 to 25 , scores of 22-25 indicate normal erectile function whereas scores of 21 or below indicate ED (Rosen et al., 1999 Rosen et al., 1999).

Penile echo-colour-Doppler was performed in basal conditions and after intracavernous injection of $10 \mu \mathrm{g}$ prostaglandin E1 (PgE1) (Aversa et al. 2005), and the peak systolic velocity (PSV) and end-diastolic velocity (EDV) were recorded at 5, 10, 15, 20 and 25 min after the injection in the proximal portion of the penis. Patients were classified as 'non-arteriogenic' when their PSV was $\geq 35 \mathrm{~cm} / \mathrm{sec}$, or $\leq 35 \mathrm{~cm} / \mathrm{sec}$, but $>25 \mathrm{~cm} / \mathrm{sec}$ with concomitant $\mathrm{EDV} \leq 0 \mathrm{~cm} / \mathrm{sec}$, and 'arteriogenic' when their PSV was $\leq 20 \mathrm{~cm} / \mathrm{sec}$ (Barassi A et al., 2009; Barassi et al., 2010). The erection quality was estimated $20 \mathrm{~min}$ after each injection. If a patient appeared stressed, he was given a second injection of the same dose of PgE1 and all measurements were repeated. From 30 to $60 \mathrm{~min}$ after the penile echo-colour-Doppler, participants were placed in a supine position and blood samples were drawn from a cubital vein into EDTA tubes. Samples were centrifuged at $4000 \mathrm{~g}$ for $10 \mathrm{~min}$. The serum was separated and stored at $-80{ }^{\circ} \mathrm{C}$ until analysis.

\section{Patients and control group}

Of a series of 590 filed ED cases studied in the period October 2008-August 2010, we randomly selected 29 arteriogenic $\mathrm{ED}$ patients (ED $\mathrm{AR}$ ) and 32 non-arteriogenic ED patients (ED NON-AR), according to our exclusion criteria for this study, for a total of 61 cases (median age 48.3 years, range 36-53) with a history of at least 3 months, but not more than 1 year of ED. General exclusion criteria were: age below 30 years, congestive heart failure, diabetes, renal failure, anaemia, acute infection or rheumatic disease, evidence from the patient's clinical history of coronary artery disease, hypertension (>140/90 $\mathrm{mm} \mathrm{Hg}$ in three consecutive recondings at rest), malignancy, systemic inflammatory disease, hepatopathies or arrhythmias, current smoking (Tostes et al., 
2008) and vitamins supplementation. Our patients did not take any therapy.

The 30 healthy controls were all blood donors (median age 45.1 years, range 34-49), and, according to their answers to the first five IIEF-15 questions (erectile response domain), did not suffer from ED. Both healthy controls and the patients of the two ED subgroups declared to develop a normal physical activity.

In accordance with the Declaration of Helsinki II, the design of the study was explained thoroughly to all the participants, and informed consent was obtained for all the test of the study from all ED patients and controls, and for administering the IIEF-15 test to controls. In particular, it was explained to the participants that no additional blood needed for this study.

\section{Assays}

From 60 to 90 min before the penile echo-color-Doppler, participants to the study were placed in a supine position and blood samples were drawn from a cubital vein into EDTA tubes. HbA1c was measured using a high performance liquid chromatography (HPLC) method (BioRad). Plasma glucose, creatinine, urea, total cholesterol, HDL-cholesterol, triglycerides, hsCRP, ALT, AST, prolactin, testosterone and $17-\beta$-estradiol were analysed with commercially available kits using Modular EVO (Roche). Plasma LDL-cholesterol was calculated with the use of Friedewald's formula. Urinary microalbumin concentration was measured in duplicate in the morning samples on a Behring Nephelometer II analyser (Siemens).

Plasma concentrations of ADMA, SDMA and L-arginine were determined by the method described by Paroni et al. (Paroni et al., 2005). Briefly, after adding L-homoarginine as internal standard, $100 \mu \mathrm{L}$ of plasma samples were submitted to solid phase extraction on SCX cartridges (Phenomenex STRATA SCX, $55 \mu \mathrm{m}, 70$ A, $100 \mathrm{mg} / 1 \mathrm{~mL})$. Following three washing steps with TCA 2\%, phosphate buffer $\mathrm{pH} 8.0$ and methanol, respectively, cationic amino acids (ADMA, SDMA, homoarginine and L-arginine) were eluted with $1 \mathrm{~mL}$ of a methanol : water solution $(70: 30, \mathrm{v}: \mathrm{v})$ containing $2 \%$ triethylamine. The eluate was evaporated to dryness under a gentle nitrogen stream and the residue was resuspendend in water. This was followed by online ortho-phthaldialdehyde derivatisation, HPLC separation (Ultrasphere Beckman ODS, $250 \times 4.6 \mathrm{~mm}, 5 \mu \mathrm{m})$ and fluorimetric detection $\left(\lambda_{\text {ecc }}\right.$ $\left.340 \mathrm{~nm}, \lambda_{\mathrm{em}} 455 \mathrm{~nm}\right)$. The liquid chromatographic system included an Agilent 1200 Series Rapid Resolution chromatograph equipped with binary pump SL, thermostatted column compartment SL, high performance autosampler SL plus and a 1260 Infinity Fluorescence Detector. Within-assay and between-assay variations for all the analytes were $<3.0 \%$ and $<6.0 \%$, respectively; the detection limit of the assay was 1.5 pmol injected at a signal-to-noise ratio of $3: 1$. The total run time was $32 \mathrm{~min}$.

\section{Statistical analysis}

All the variables were expressed as median and range (or $25 \%$ and $75 \%$ percentiles). Normal distribution of the data was tested by the Kolmogorov-Smirnov test. The significance of differences between controls, ED AR and ED non-AR was assessed by the non-parametric test Mann-Whitney Rank sum test. Spearman correlation was used to assess the strength of association between variables. Probabilities $\leq 0.05$ were considered to be statistically significant. All analyses were performed using the statistical software package Sigma Stat (Statistical Analysis System, version 3.0) (Jandel Scientific GmbH, Erkrath, Germany).

\section{Results}

Of the 61 men included in the study, 29 (47.5\%) were classified as having ED AR and 32 (52.5\%) as having ED NON-AR. Clinical and laboratory features of 30 controls and 61 patients with ED are presented in Table 1. In ED AR (median age 49.2 years, range $42-52$ ) and ED NONAR (median age 47.5 years, range 36-53) the median of IIEF values were 10.5 (range 5-19) and 12.8 (range 7-20) respectively. There were no significant differences between the two ED subgroups $(p>0.05)$. There were no differences neither between ED patients and controls nor between the two ED subgroups regarding all the laboratory tests $(p>0.05)$. Hormonal status of all patients was in the normal range (Table 1$)$.

The ADMA concentration (median and range in $\mu \mathrm{mol} / \mathrm{L})$ in $\mathrm{ED} \mathrm{AR}[0.682(0.536-0.811)]$ and in $\mathrm{ED}$ NON-AR [0.554 (0.492-0.722)] was significantly higher $(p=0.003)$ compared with controls $[0.445 \quad(0.380-$ $0.570)]$; in ED AR it was also significantly higher $(p=0.038)$ than in ED NON-AR.

The SDMA concentration in ED AR [0.746 (0.613$0.808)]$ and in ED NON-AR [0.592 (0.528-0.751)] was significantly higher ( $p=0.003$ and $p \leq 0.001$ respectively) compared with controls [0.465 (0.425-0.529)]; in ED AR it was also significantly higher $(p=0.030)$ than in ED NON-AR. The results are reported in Fig. 1.

Spearman correlation analysis demonstrated a negative correlation between serum ADMA levels and IIEF score that reached the significance in ED AR $(r=-0.396$; $p<0.01)$ but without reaching the significance in ED NON-AR $(r=-0.03 ; p<0.20)$.

The L-arginine plasma concentration (median and range in $\mu \mathrm{mol} / \mathrm{L})$ did not differ significantly $(p>0.05)$ 
Table 1 Clinical and laboratory features of controls and patients with erectile dysfunction

\begin{tabular}{|c|c|c|c|c|}
\hline & Controls $(n=30)$ & $\mathrm{ED}(n=61)$ & $\operatorname{ED}-\mathrm{AR}(n=29)$ & ED-NON AR $(n=32)$ \\
\hline Age (years) & $45.1(34-49)$ & $48.3(36-53)$ & $49.2(42-52)$ & $47.5(36-53)$ \\
\hline Systolic pressure (mmHg) & $125(118-137)$ & $128(116-138)$ & $130(118-138)$ & $127(116-135)$ \\
\hline Diastolic pressure $(\mathrm{mmHg})$ & $82(75-86)$ & $85(72-88)$ & $87(80-88)$ & $83(72-86)$ \\
\hline Heart rate (bpm) & $72(65-81)$ & $75(63-88)$ & $77(65-88)$ & $73(63-85)$ \\
\hline Body mass index $\left(\mathrm{kg} / \mathrm{m}^{2}\right)$ & $26.2(22.3-27.4)$ & $27.3(21.8-28.2)$ & $27.5(22.5-28.2)$ & $26.8(21.8-27.7)$ \\
\hline Diuresis (L) & $1.6(1.3-2.0)$ & $1.7(1.2-2.1)$ & $1.8(1.4-2.3)$ & $1.5(1.2-1.99)$ \\
\hline Microalbuminuria (mg/L) & $11(11-14)$ & $11(11-13)$ & $11(11-13)$ & $11(11-12)$ \\
\hline $\mathrm{HbA}_{1 \mathrm{c}}(\%)$ & $4.9(4.7-5.2)$ & $5.2(5.0-5.4)$ & $5.2(4.9-5.3)$ & $5.2(5.0-5.4)$ \\
\hline Glucose (mg/dL) & $90.2(80-96)$ & 94.1 (85-99) & $95.9(89-102)$ & $93.3(84-95)$ \\
\hline hs-PCR (mg/L) & $0.9(0.5-1.9)$ & $1.1(0.6-2.1)$ & $1.3(0.7-2.5)$ & $0.8(0.5-1.5)$ \\
\hline 17- $\beta$-estradiol $(\mathrm{pg} / \mathrm{mL})$ & $26(22.5-27.8)$ & $25.5(22.5-31.4)$ & $25.8(20.0-33.5)$ & $25.5(23.8-30.5)$ \\
\hline Prolactin $(\mathrm{ng} / \mathrm{mL})$ & $6.9(5.8-8.5)$ & $7.5(6.1-9.1)$ & $7.4(6.6-8.4)$ & $7.9(6.0-9.9)$ \\
\hline Testosterone $(\mathrm{ng} / \mathrm{mL})$ & $4.9(3.9-5.3)$ & $4.6(3.8-5.1)$ & $4.5(3.8-4.8)$ & $4.7(3.8-5.5)$ \\
\hline Creatinine (mg/dL) & $0.81(0.75-0.88)$ & $0.85(0.79-1.00)$ & $0.82(0.73-0.89)$ & $0.85(0.78-0.92)$ \\
\hline Urea (mg/dL) & $37(32-45)$ & $38(33-45)$ & $37(32-46)$ & $39(34-45)$ \\
\hline $\operatorname{ALT}(\mathrm{U} / \mathrm{L})$ & $25(20-32)$ & $26(19-39)$ & $29(22-43)$ & $26(19-34)$ \\
\hline AST $(U / L)$ & $28(20-34)$ & $27(22-31)$ & $29(23-32)$ & $26(22-31)$ \\
\hline Total cholesterol (mg/dL) & $196(159-220)$ & $208(177-226)$ & $198(175-213)$ & $211(186-227)$ \\
\hline Triglycerides (mg/dL) & $118(92-140)$ & $112(89-144)$ & $116(94-161)$ & $112(84-131)$ \\
\hline HDL cholesterol (mg/dL) & $53(45-63)$ & $55(46-64)$ & $52(46-61)$ & $57(50-64)$ \\
\hline IIEF-5 score & $23(22-25)$ & $11.6(5-20)^{a}$ & $10.5(5-19)^{a}$ & $12.8(7-20)^{a}$ \\
\hline
\end{tabular}

Values are reported as median and range.

${ }^{a} p<0.001$ vs. Controls.

neither between ED AR [54.10 (46.67-65.57)] or between ED NON-AR [53.80 (40.23-62.30)] and controls [47.15 (36.20-60.50)] nor between the two ED subgroups.

The L-arginine/ADMA ratio [66.50 (52.17-90.95)] and L-arginine/SDMA ratio [72.10 (59.37-82.7)] in ED AR were significantly lower $(p=0.002)$ than in controls $[100$ (85.5-136.5) and $105.5(78-144)]$ and than in ED NONAR $[87.70(82.40-116.70) p=0.004$ and $82.90(78-114)$ $p=0.029]$; the two rations in ED NON-AR did not differ $(p>0.05)$ from the controls (Fig. 2).

\section{Discussion and conclusions}

Cells regulate NO homeostasis by different mechanisms. eNOS (type 3) and nNOS (type 1) are constitutively expressed low output enzymes whose ezymatic activity depends on $\mathrm{Ca}^{2}+$ levels, protein myristilation, phosphorylation and protein/protein interactions. By contrast, iNOS (type 2) is an inducible NO synthase not normally expressed in resting cells, and upon stimulation by various cytokines or bacterial lipopolysaccharide its transcription can be induced within several hours. The discovery of ADMA and demonstration of in vivo and in vitro effects on inhibiting NO synthesis has led to a large body of work attempting to discover its role in a wide range of different clinical conditions which share endothelial dysfunction as a common feature. After proteolysis of methylated proteins, ADMA is released within cells where can concur to regulate excessive iNOS activity, as well as the other NOS isoforms by competing with the NOS natural substrate arginine (Vallance et al., 1992; Ueda et al., 2003). The local concentrations of substrate and inhibitors, i.e. the L-arginine/ADMA concentration ratio, may be therefore an important factor, although not the only, in the pathogenesis of ED in various disease states. Presumably, the state of activation or inhibition of NOS will depend on ADMA exported from the site of origin by cationic amino acid transporter, the same used by L-arginine (CAT) (Baylis 2006; Welch et al., 1997). The majority of ADMA is metabolized by the enzyme DDAH to yield citrulline and dimethyl-amine (Achan et al., 2003; Tsuji et al., 1986; Palm et al., 2007). Intracellular ADMA that escapes metabolism by DDAH, besides to inhibit NOS, may also reduce NO generation by competing with L-arginine for cellular uptake by CAT transporter. Thus, ADMA not only blocks NOS activity but also limits the cellular uptake of L-arginine, thereby contributing to oxidative stress that further inhibits NO biogenesis (Bögerk et al., 2009). SDMA, in contrast, has no direct effect on NOS. However, it may still be important by competing with L-arginine for transport across the CAT (Closs et al., 1997; Bode- Böger et al., 2006). Thus, also SDMA may indirectly limit NO generation.

The populations studied, ED and controls, were homogeneous with similar patterns of metabolic and inflammatory variables. It is generally accepted that ED has been 

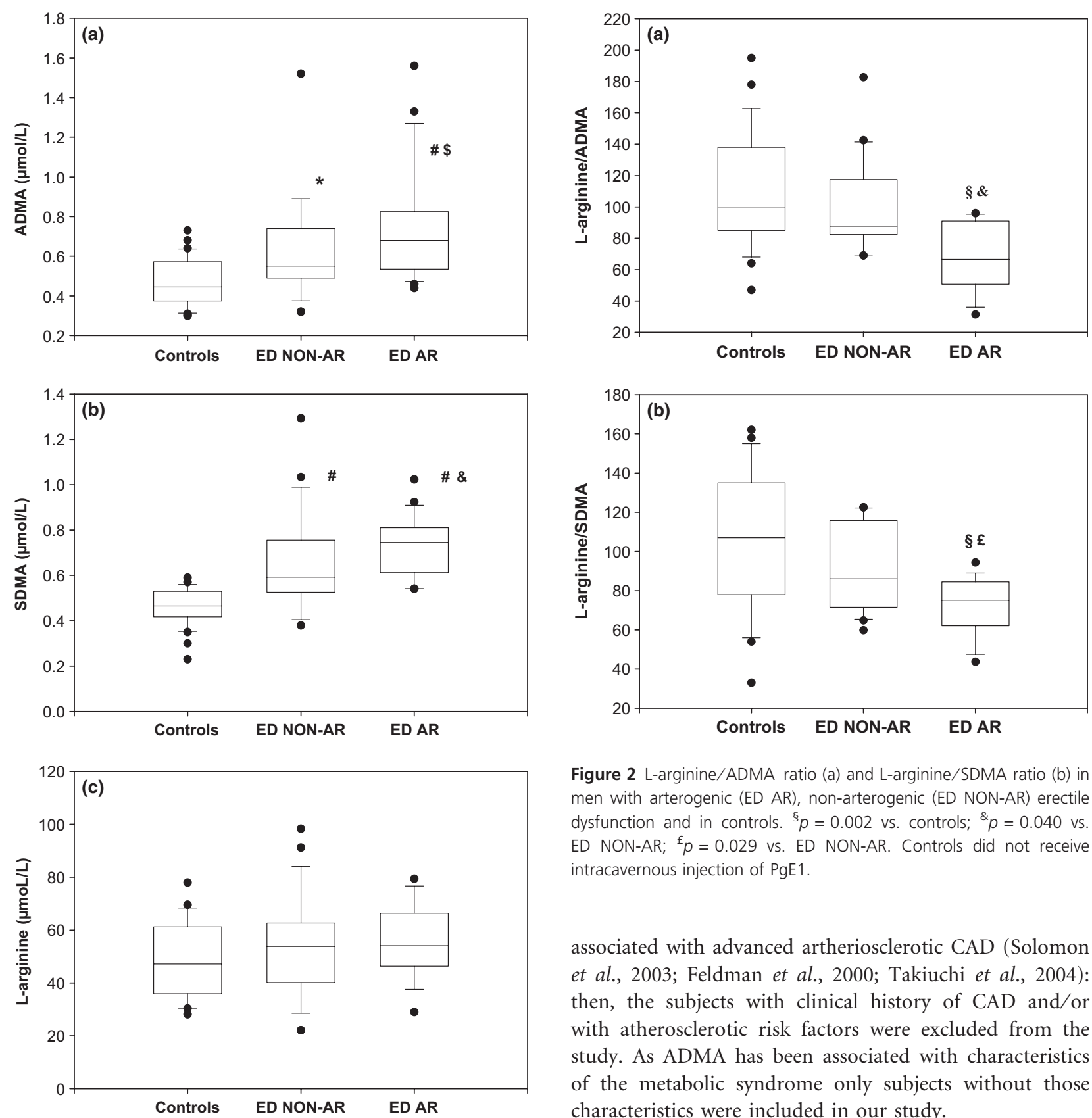

Figure 1 Plasma ADMA (a), SDMA (b) and L-arginine (c) concentrations in men with arterogenic (ED AR), non-arterogenic (ED NON-AR) erectile dysfunction and in controls. ${ }^{*} p=0.003$ vs. controls; ${ }^{\#} p<0.001$ vs. controls; ${ }^{\$} p=0.038$ vs. ED NON-AR; ${ }^{\&} p=0.030$ vs. ED NON-AR. Controls did not received intracavernous injection of PgE1. The boundary of the box closest to zero indicates the 25th percentile, the line within the box marks the median, and the boundary of the box farthest from zero indicates the 75th percentile. Whiskers (error bars) above and below the box indicate the 90th and 10th percentiles. Black circles are the outlying points.

Figure 2 L-arginine/ADMA ratio (a) and L-arginine/SDMA ratio (b) in men with arterogenic (ED AR), non-arterogenic (ED NON-AR) erectile dysfunction and in controls. ${ }^{\S} p=0.002$ vs. controls; ${ }^{\&} p=0.040$ vs. ED NON-AR; ${ }^{f} p=0.029$ vs. ED NON-AR. Controls did not receive intracavernous injection of PgE1.

associated with advanced artheriosclerotic CAD (Solomon et al., 2003; Feldman et al., 2000; Takiuchi et al., 2004): then, the subjects with clinical history of CAD and/or with atherosclerotic risk factors were excluded from the study. As ADMA has been associated with characteristics of the metabolic syndrome only subjects without those characteristics were included in our study.

In this study of well-characterized patients, in particular as regards the penile arteriogenic and non-arteriogenic aetiology of $\mathrm{ED}$, we found a significant increase of ADMA and SDMA concentrations in both the ED subgroups compared with healthy controls and in ED AR compared with ED NON-AR. Our findings confirm recent data of Ioakeimidis et al. that found ADMA levels significantly higher in ED AR than in ED NON-AR patients with an independent inverse association between ADMA and PSV (Ioakeimidis et al. 2011).

Although elevated ADMA levels in patients with renal failure could be attributed, at least in part, to reduced 
renal excretion, the precise cause of elevated ADMA levels in ED has not been identified yet. One cause could be a reduced activity of DDHA, highly expressed in the liver (Nijveldt et al., 2003), whose activity may be inhibited by a large series of traditional (hypercholesterolaemia, diabetes, smoking), non-traditional (hyperhomocysteinaemia, inflammation, oestrogen deficiency, insulin resistence, hypothyroidism) risk factors and finally by oxidative stress. A fairly recent study showed that plasma reactive oxygen metabolite (ROS) concentrations were higher and plasma total antioxidant status (TAS) was lower in ED AR patients in comparison with ED NON-AR patients and in controls (Barassi et al., 2009). Furthermore, through uncoupling of NOS, increased production of superoxide could further impair DDAH, leading to a perpetuating increase in ADMA concentration (Sydow et al., 2003).

There were no significant differences neither between ED patients and controls nor between the two ED subgroups regarding L-arginine concentration. L-arginine/ADMA ratio and L-arginine/SDMA ratio were significantly lower in ED AR in comparison with those in the controls and in ED NON-AR. In ED NON-AR, the two ratios were not different from those in the controls. A low L-arginine/ADMA ratio (i.e. the ratio of NOS substrate and its endogenous inhibitor concentrations) is compatible with ADMA acting as a competitive inhibitor of NOS, resulting in a reduced NO synthesis. It is more difficult to explain the increased level of SDMA and the decreased L-arginine/SDMA ratio in ED patients. In fact, the role of SDMA in these pathologies is not yet completely clear. Nevertheless, the decrease of the ratio only in $\mathrm{ED}$ AR can contribute to limit NO generation by reducing the availability of L-arginine, the NOS substrate.

The HPLC method used for quantification is characterized by a very low imprecision, allowing reliable determination also in the presence of small concentration differences between groups. Nevertheless, we found near borderline significant differences in ADMA and L-arginine/ADMA levels between $\mathrm{AR}$ and NON-AR patients; then, from a diagnostic standpoint it needs caution in discerning between AR and NON-AR patients from levels near the limit of reference ranges. The concentrations of both ADMA and SDMA are tightly controlled in health, with intraindividual variations $\left(\mathrm{CV}_{\mathrm{i}}\right)$ of $7.4 \%$ and $5.8 \%$ respectively (Blackwell et al., 2007). Based on a desirable imprecision of no more than 0.5 times $\mathrm{CV}_{\mathrm{i}}$ (Fraser et al., 1999), our method meet this goal. In addition, both the generation of $\mathrm{NO}$ and the formation of the $\mathrm{L}$-arginine analogous, by the sequence of protein methylation and proteolysis, are intracellular processes. ADMA and SDMA in plasma probably originate from cellular spillover. A limited increase in the plasma concentration may thus reflect a much larger increase of their intracellular concentration, which may be sufficient to substantially inhibit NO production. In fact, cells can concentrate L-arginine analogous up to 5-10 times than that in plasma.

This study supports the hypothesis that elevated ADMA and normal-low or low L-arginine levels may contribute, either directly or through NOS uncoupling, to the inhibition of penile NO synthesis (Maas et al., 2002). In fact, also L-arginine/ADMA ratio in $\mathrm{ED}$ AR is significantly lower than in ED NON-AR and controls. Furthermore, the increased SDMA competes with the L-arginine entrance in the cells reducing the availability for NO synthesis. ED, but only ED AR, may be the earliest manifestation of a generalized vascular disease and only these patients may be at risk of later developing CAD.

Recently, Elesber (Elesber et al., 2006) and Aktoz (Aktoz et al., 2010), comparing plasma ADMA concentration and severity of ED and coronary artery disease, demonstrated a significantly negative correlation between IIEF score and ADMA levels, but none of the two studies split the ED patients in relation to the different aetiology (AR and NON-AR) according to the penile echo-colourDoppler after intracavernous injection of prostaglandin E1. Our results showed a significantly negative correlation between serum ADMA levels and IIEF score but only in ED AR subgroup confirming the role of ADMA in ED but only in those patients with ED of arteriogenic origin.

Chen et al. (Chen et al., 1999) reported significant improvement in sexual function when ED patients characterized by relative L-arginine deficiency (L-arginine/ADMA elevated), were treated with L-arginine supplementation in combination with phosphodiesterase type s (PDE-5) inhibitors. Quite recently, the oral supplementation with arginine or citrulline (which escapes intestinal or liver metabolism, enters the kidneys and is rapidly converted into L-arginine) has been also proposed to patients with mild ED and in heart failure (Cormio et al., 2011; Orozco-Gutierrez et al., 2010), thus providing the rationale for oral L-citrulline supplementation as a donor for the L-arginine/NO pathway in patients with endothelial dysfunction. From the results of our study, we can speculate that only a subgroup of ED patients, ED AR with elevated ADMA, might benefit from L-arginine or L-citrulline supplementation. For this reason, we think advisable to recognize between the ED patients those with arteriogenic aetiology.

In conclusion, NO pathway is of critical importance in the physiological regulation of erection. Elevated endogenous NOS inhibitor, like ADMA, not balanced by and increased L-arginine concentration, contributes to $\mathrm{NO}$ deficiency, leading impaired NO-mediated relaxation in the lower urinary tracts and corpus cavernosum. Moreover, our observations could be also interpreted as 
evidence for a reduced systemic availability of L-arginine in men with ED; but only in men with ED AR, ADMA appears to be preferentially elevated and both L-arginine/ADMA ratio and L-arginine/SDMA ratio decreased; thus, confirming in this subgroup the diagnosis of vascular origin of ED obtained with the penile echo-colourDoppler procedure. Nevertheless, the levels of ADMA and of L-arginine/ADMA are not sufficiently different to accurately discern between $\mathrm{AR}$ and NON-AR patients. Probably because the precise aetiology of NON-AR ED can be difficult to elucidate and may be because of a combination of organic as well as psychological factors. Taken together with the relatively small number of samples in this study, this may account for the fact that levels of ADMA and L-arginine/ADMA, even though a statistically significant difference was shown between AR and NON-AR patients, can not accurately differentiate a single patient presenting with ED in clinical setting.

Additional studies are required to define the importance of ADMA in ED, but the results demonstrate that future research should investigate separately AR from ED NON-AR patients, because they belongs to different population and accordinges to severity of arteriogenic status by using PSV values (Ioakeimidis et al., 2011). This will increase understanding of the role of ADMA in the mechanisms that lead to ED and potentially provide therapeutic targets.

Finally, our results point out the importance of the determination of ADMA, SDMA and L-arginine concentration that we suggest to insert in the list of the tests for an early screening of ED patients.

\section{References}

Achan V, Broadhead M, Malaki M, Whitley G, Leiper J, MacAllister R \& Vallance P. (2003) Asymmetric dimethylarginine causes hypertension and cardiac dysfunction in humans and is actively metabolized by dimethylarginine dimethylaminohydrolase. Arterioscler Thromb Vasc Biol 23, 1455-1459.

Aktoz T, Aktoz M, Tatlı E, Kaplan M, Turan FN, Barutcu A, Atakan IH, Demir M \& Altun A. (2010) Assessment of the relationship between asymmetric dimethylarginine and severity of erectile dysfunction and coronary artery disease. Int Urol Nephrol 42, 873-879.

Aversa A, Bruzziches R \& Spera G. (2005) Diagnosing erectile dysfunction: the penile dynamic colour duplex ultrasound revisited. Int $J$ Androl 28(Suppl 2), 61-63.

Baylis C. (2006) Arginine, arginine analogs and nitric oxide production in chronic kidney disease. Nat Clin Pract Nephrol 2, 209-220.

Barassi A, Colpi GM, Piediferro G, Dogliotti G, Melzi d'Eril GV \& Corsi MM. (2009) Oxidative stress and antioxidant status in patients with erectile dysfunction. J Sex Med 6, 2820-2825.

Barassi A, Pezzilli R, Morselli-Labate AM, Porreca W, Piediferro G, Ciociola F, Colpi G \& Melzi d'Eril GV. (2010) Evaluation of microalbuminuria in patients with erectile dysfunction. J Sex Med 7, 1224-1228.
Benet AE \& Melman A. (1995) The epidemiology of erectile dysfunction. Urol Clin North Am 22, 699-709.

Blackwell S, O'Reilly DS \& Talwar D. (2007) Biological variation of asymmetric dimethylarginine and related arginine metabolites and analytical performance goals for their measurement in human plasma. Eur J Clin Invest 37, 364-371.

Bode-Böger SM, Scalera F, Kielstein JT, Martens-Lobenhoffer J, Breithardt G, Fobker M \& Reinecke H. (2006) Symmetrical dimethylarginine: a new combined parameter for renal function and extent of coronary artery disease. J Am Soc Nephrol 17, 1128-1134.

Bögerk RH, Maask R, Schulzek F \& Schwedhelmk E. (2009) Asymmetric dimethylarginine (ADMA) as a prospective marker of cardiovascular disease and mortality-an update on patient populations with a wide range of cardiovascular risk. Pharmacol Res 60, 481-487.

Burchardt M, Burchardt T, Anastasiadis AG, Kiss AJ, Shabsigh A, de La Taille A, Pawar RV, Baer L \& Shabsigh R. (2001) Erectile dysfunction is a marker for cardiovascular complications and psychological functioning in men with hypertension. Int J Impot Res 13, 276-281.

Carrier S, Hricak H, Lee S, Baba K, Morgan DM, Nunes L, Rosa GY, Phillips TL \& Lue TF. (1995) Radiation-induced decrease in nitric oxide synthase-containing nerves in the rat penis. Radiology 195 , 95-99.

Chen J, Wollman Y, Chernichovsky T, Iaina A, Sofer M \& Matzkin H. (1999) Effect of oral administration of high-dose nitric oxide donor L-arginine in men with organic erectile dysfunction: results of a double-blind, randomized, placebo-controlled study. BJU Int 83, 269-273.

Closs EI, Basha FZ, Habermeier A \& Förstermann U. (1997) Interference of L-arginine analogues with L-arginine transport mediated by the $\mathrm{y}+$ carrier hCAT-2B. Nitric Oxide 1, 65-73.

Cooke JP. (2004) Asymmetrical dimethylarginine: the Uber marker? Circulation 109, 1813-1818.

Cormio L, De Siati M, Lorusso F, Selvaggio O, Mirabella L, Sanguedolce F \& Carrieri G. (2011) Oral L-citrulline supplementation improves erection hardness in men with mild erectile dysfunction. Urology 77, 119-122.

De Angelis L, Marfella MA, Siniscalchi M, Marino L, Nappo F, Giugliano F, De Lucia D \& Giugliano D. (2001) Erectile and endothelial dysfunction in type II diabetes: a possible link. Diabetologia 44, 1155-1160.

Elesber AA, Solomon H, Lennon RJ, Mathew V, Prasad A, Pumper G, Nelson RE, McConnell JP, Lerman LO \& Lerman A. (2006) Coronary endothelial dysfunction is associated with erectile dysfunction and elevated asymmetric dimethylarginine in patients with early atherosclerosis. Eur Heart J 27, 824-831.

Feldman HA, Johannes CB, Derby CA, Kleinman KP, Mohr BA, Araujo AB \& McKinlay JB. (2000) Erectile dysfunction and coronary risk factors: prospective results from the Massachusetts male aging study. Prev Med 30, 328-338.

Fraser CG \& Petersen PH. (1999) Analytical performance characteristics should be judged against objective quality specifications. Clin Chem 45, 321-323.

Garban H, Vernet D, Freedman A, Rajfer J \& Gonzalez-Cadavid NF. (1995) Effect of aging on nitric oxide mediated penile erection in rats. Amer J Physiol 268, H467-475.

Hashimoto M, Kozaki K, Eto M, Akishita M, Ako J, Iijima K, Kim S, Toba K, Yoshizumi M \& Ouchi Y. (2000) Association of coronary risk factors and endothelium-dependent flow-mediated dilatation of the brachial artery. Hypertens Res 23, 233-238. 
Ioakeimidis N, Vlachopoulos C, Rokkas K, Aggelis A, Terentes-Printzios D, Samentzas A, Alexopoulos N \& Stefanadis C. (2011) Relationship of asymmetric dimethylarginine with penile Doppler ultrasound parameters in men with vasculogenic erectile dysfunction. Eur Urol 59, 948-955.

Kendirci M, Trost L, Sikka S \& Hellstrom WJ. (2007) The effect of vascular risk factors on penile vascular status in men with erectile dysfunction. J Urol 178, 2516-2520.

Kielstein JT \& Zoccali C. (2005) Asymmetric dimethylarginine: a cardiovascular risk factor and a uremic toxin coming of age? Am J Kidney Dis 46, 186-202.

Lugg JA, Rajfer J \& Gonzalez-Cedavid NF. (1995) Dihydrotestosterone is the active androgen in the maintenance of nitric oxide-mediated penile erection in the rat. Endocrinology 136, 1495-1501.

Maas R, Schwedhelm E, Albsmeier J \& Böger RH. (2002) The pathophysiology of erectile dysfunction related to endothelial dysfunction and mediators of vascular function. Vasc Med 7, 213-225.

Maas R, Wenske S, Zabel M, Ventura R, Schwedhelm E, Steenpaß A, Klemm H, Noldus J \& Boger RH. (2005) Elevation of asymmetrical dimethylarginine (ADMA) and coronary artery disease in men with erectile dysfunction. Eur Urol 48, 1004-1012.

Nijveldt RJ, Teerlink T, Siroen MP, van Lambalgen AA, Rauwerda JA \& van Leeuwen PA. (2003) The liver is an important organ in the metabolism of asymmetrical dimethylarginine (ADMA). Clin Nutr $22,17-22$.

Orozco-Gutiérrez JJ, Castillo-Martínez L, Orea-Tejeda A, Vázquez-Díaz O, Valdespino-Trejo A, Narváez-David R, Keirns-Davis C, CarrascoOrtiz O, Navarro-Navarro A \& Sánchez-Santillán R. (2010) Effect of L-arginine or L-citrulline oral supplementation on blood pressure and right ventricular function in heart failure patients with preserved ejection fraction. Cardiol J 17, 612-618.

Palm F, Onozato ML, Luo Z \& Wilcox CS. (2007) Dimethylarginine dimethylaminohydrolase (DDAH): expression, regulation, and function in the cardiovascular and renal systems. Am J Physiol Heart Circ Physiol 293, H3227-H3245.

Palmer RM, Ashton DS \& Moncada S. (1988) Vascular endothelial cells synthesize nitric oxide from L-arginine. Nature 333, 664-666.

Paroni R, Fermo I, Fiorina P \& Cighetti G. (2005) Determination of asymmetric and symmetric dimethylarginines in plasma of hyperhomocysteinemic subjects. Amino Acids 28, 389-394.

Rosen RC, Cappelleri JC, Smith MD, Lipsky J \& Peña BM. (1999) Development and evaluation of an abridged, 5-item version of the International Index of Erectile Function (IIEF-5) as a diagnostic tool for erectile dysfunction. Int J Impot Res 11, 319-326.

Rosen RC, Riley A, Wagner G, Osterloh IH, Kirkpatrick J \& Mishra A. (1997) The international index of erectile function (IIEF): a multidimensional scale for assessment of erectile dysfunction. Urology 49, 822-830.
Sáenz de Tejada I, Angulo J, Cellek S, González-Cadavid N, Heaton J, Pickard R \& Simonsen U. (2004) Physiology of erectile function. J Sex Med 1, 254-265.

Schiel R, Franke S, Busch M, Müller A, Fleck C, Müller UA, Braun A \& Stein G. (2003) Effect of smoking on risk factors for cardiovascular disease in patients with diabetes mellitus and renal insufficiency. Eur J Med Res 8, 283-291.

Sydow K \& Münzel T. (2003) ADMA and oxidative stress. Atheroscler Suppl 4, 41-51.

Solomon H, Man JW \& Jackson G. (2003) Erectile dysfunction and the cardiovascular patient: endothelial dysfunction is the common denominator. Heart 89, 251-253.

Solomon H, Man JW, Wierzbicki AS \& Jackson G. (2003) Relation of erectile dysfunction to angiographic coronary artery disease. Am J Cardiol 91, 230-231.

Takiuchi S, Fujii H, Kamide K, Horio T, Nakatani S, Hiuge A, Rakugi H, Ogihara T \& Kawano Y. (2004) Plasma asymmetric dimethylarginine and coronary and peripheral endothelial dysfunction in hypertensive patients. Am J Hypertens 17, 802-808.

Tostes RC, Carneiro FS, Lee AJ, Giachini FR, Leite R, Osawa Y \& Webb RC. (2008) Cigarette smoking and erectile dysfunction: focus on NO bioavailability and ROS generation. J Sex Med 5, 1284-1295.

Tsuji H, Ogawa T, Bando N \& Sasaoka K. (1986) Purification and properties of 4-aminobenzoate hydroxylase, a new monooxygenase from Agaricus bisporus. J Biol Chem 261, 13203-13209.

Ueda S, Kato S, Matsuoka H, Kimoto M, Okuda S, Morimatsu M \& Imaizumi T. (2003) Regulation of cytokine-induced nitric oxide synthesis by asymmetric dimethylarginine: role of dimethylarginine dimethylaminohydrolase. Circ Res 92, 226-233.

Valkonen VP, Päivä H, Salonen JT, Lakka TA, Lehtimäki T, Laakso J \& Laaksonen R. (2001) Risk of acute coronary events and serum concentration of asymmetrical dimethylarginine. Lancet 358, 2127-2128.

Vallance P, Leone A, Calver A, Collier J \& Moncada S. (1992) Accumulation of an endogenous inhibitor of nitric oxide synthesis in chronic renal failure. Lancet 339, 572-575.

Vallance P, Leone A, Calver A, Collier J \& Moncada S. (1992) Endogenous dimethylarginine as an inhibitor of nitric oxide synthesis. J Cardiovasc Pharmacol 20(Suppl 12), S60-S62.

Welch WJ \& Wilcox CS. (1997) Macula densa arginine delivery and uptake in the rat regulates glomerular capillary pressure. Effects of salt intake. J Clin Invest 100, 2235-2242.

Widlansky ME, Gokce N, Keaney JF Jr \& Vita JA. (2003) The clinical implications of endothelial dysfunction. J Am Coll Cardiol 42, 11491160.

Wierzbicki AS, Solomon H, Lumb PJ, Lyttle K, Lambert-Hammill M \& Jackson G. (2006) Asymmetric dimethyl arginine levels correlate with cardiovascular risk factors in patients with erectile dysfunction. Atherosclerosis 185, 421-425. 